Service social

\title{
Conscientisation et santé mentale : une pratique en région
}

\section{Robert Arsenault}

Volume 40, numéro 3, 1991

Oppression, intolérance et intervention

URI : https://id.erudit.org/iderudit/706544ar

DOI : https://doi.org/10.7202/706544ar

Aller au sommaire du numéro

Éditeur(s)

École de service social de l'Université Laval

ISSN

1708-1734 (numérique)

Découvrir la revue

Citer cet article

Arsenault, R. (1991). Conscientisation et santé mentale : une pratique en région. Service social, 40(3), 53-67. https://doi.org/10.7202/706544ar

\section{Résumé de l'article}

Cet article vient rendre compte de deux expériences en intervention de groupe réalisées dans le cadre d'un programme de réinsertion sociale (case management) mis sur pied par le Centre hospitalier Baie-des-Chaleurs de Maria.

L'approche utilisée dans ces interventions s'est inspirée largement du modèle de conscientisation élaboré par Paulo Freire pour l'alphabétisation des masses populaires au Brésil au début des années 60.

Cette approche mise sur le dialogue comme moteur d'une prise en charge individuelle et collective des problèmes qui nous concernent. Elle peut également être une base de réflexion pour amorcer une critique du discours institutionnel qui a pour effet la reproduction des inégalités sociales. 
Robert Arsenault, agent de réinsertion sociale, Centre hospitalier Baiedes-Chaleurs de Maria.

\section{Conscientisation et santé mentale : une pratique en région}

Depuis quatre ans se vit dans la Baie-des-Chaleurs une expérience d'intervention avec des personnes subissant une forme particulière d'oppression liée à leur condition de santé. Il s'agit du développement d'une approche conscientisante avec des personnes atteintes de maladie mentale sévère (psychose, schizophrénie) ainsi qu'avec un groupe de leurs parents'.

Dans un premier temps, je tâcherai d'illustrer le vécu de ces personnes et de faire ressortir les aspects particuliers à une région comme la nôtre en tâchant de mettre en relief les forces et les faiblesses d'un tel milieu. Il sera intéressant de parler des capacités d'accueil de la maladie mentale de la part des gens qui y vivent ainsi que de leurs limites ou résistances à se laisser trop approcher par ces personnes en difficulté.

Ce texte tentera de faire ressortir la dynamique d'oppression vécue par les personnes aux prises avec la maladie mentale dans le contexte structurel dans lequel elles sont appelées à vivre. Je veux parler ici de la situation précaire de l'emploi, du vécu avec l'aide sociale, du pouvoir institutionnel, etc.

La deuxième partie de cet article viendra présenter brièvement les principaux éléments composant une démarche qui se veut conscientisante. Y seront abordés certains concepts utilisés par Paulo Freire $^{2}$ qui nous serviront d'éléments de base dans la compréhension de cette approche.

En troisième lieu, j'expliquerai comment, dans la pratique, nous avons appliqué ces concepts. Je parlerai d'abord du contexte dans lequel est née cette démarche pour parler des expériences que nous poursuivons actuellement. 
II sera d'abord question du groupe de parents; je tâcherai alors d'illustrer l'amorce de la démarche, son déroulement ainsi que les étapes franchies jusqu'à ce jour. Cette illustration visera à mettre en relief les particularités d'une démarche conscientisante et à amener le lecteur à faire sa propre réflexion quant à la portée transformatrice d'une telle approche.

Je parlerai par la suite, en m'appuyant sur les mêmes principes de base, de l'expérience que nous menons avec un groupe de personnes atteintes de maladie mentale en faisant ressortir les particularités d'une démarche basée sur le dialogue avec ces personnes.

Enfin, une conclusion viendra jeter un regard critique sur nos pratiques dites institutionnelles.

\section{La problématique}

Vivre avec la maladie mentale dans la Baie-des-Chaleurs, c'est devoir se débattre avec une situation qui conduit souvent à l'isolement social, même s'il existe dans plusieurs milieux certaines capacités de tolérance, voire d'accueil envers les personnes qui sont aux prises avec ce problème.

Dans des petites communautés comme la nôtre où la densité de population est très faible ( 35000 habitants sur 225 kilomètres de côte), les gens se connaissent beaucoup; mais les préjugés à l'égard de la maladie mentale sont quand même très forts.

Si une famille se retrouve aux prises avec la maladie mentale, c'est souvent un mécanisme de rejet qui va s'installer dans son environnement social. Les amis vont s'éloigner, la parenté va se faire plutôt discrète et distante. Ce sont souvent des sentiments d'impuissance et de peur qui vont amener les personnes à s'éloigner de celles qui sont atteintes par la maladie mentale.

Pour la famille qui fait subitement face à ce problème, c'est souvent le désarroi complet. Elle ne sait pas ce qui se passe, très souvent elle ne connaît pas les ressources et se culpabilise de ce qui arrive à son enfant.

Prenons un exemple : La famille $X$ vit un peu comme toutes les autres familles dans son milieu. Les trois enfants ont toujours bien fonctionné à l'école et les parents mènent une vie qui ressemble à celle de bien d'autres parents. Cependant, Marie qui doit terminer son cégep cette année n'a pas pu finir sa session d'autonme parce que, diton, elle est en "dépression ". Mais plus le temps passe, plus elle se retire dans sa chambre; elle dit entendre des voix, et la nuit elle crie et ameute toute la maison parce que, selon elle, il y a des gens qui passent par la trappe d'aération et qui viennent la violer. Les parents ne savent 
plus quoi faire. Cela devient même très pénible de l'approcher, parce qu'elle leur dit qu'elle ne veut plus les voir.

Dans de telles circonstances, les parents vont appeler ou se rendre directement avec leur enfant à la salle d'urgence du centre hospitalier qui est à quarante kilomètres de chez eux. Une fois sur place, ils doivent remplir les formalités administratives d'usage, attendre qu'un médecin les accueille tout en tâchant d'avoir le contrôle à la fois sur leur enfant et sur eux-mêmes pour, enfin, obtenir une hospitalisation et s'en retourner souvent complètement découragés et inquiets quant à ce qui peut arriver.

Après plusieurs expériences de ce genre, certains parents en viennent à se sentir envahis par la situation. Ils ne savent plus comment ils vont s'en sortir ni comment ils vont pouvoir venir en aide vraiment à leur enfant.

L'expérience suivante a été vécue fréquemment par bien des parents de la Baie-des-Chaleurs : ils se sont rendus à l'hôpital local de courte durée, c'est-à-dire à Maria; il a pu arriver que, de là, leur enfant ait été transféré à Mont-Joli (à $300 \mathrm{~km}$ ) qui est l'hôpital régional de longue durée, d'où, au bout de deux semaines, il aura reçu son congé, parfois même sans que la famille en ait été avisée. Les parents le retrouvent alors «bourré de pilules», parfois somnolent et fonctionnant au ralenti.

Dans ces cas, la crise a été contrôlée, mais la personne ne fonctionne pas plus qu'avant et semble s'enfoncer dans un monde où personne ne peut la rejoindre.

Chez nous, certains parents vivaient cette situation depuis plusieurs années (particulièrement avant l'implantation du service de santé mentale, il y a six ans) et n'avaient jamais eu la chance d'en parler ouvertement. Dans de telles situations, l'oppression se vit au jour le jour à travers la solitude et l'isolement. "Je me croyais la seule de Matapédia à Gaspé à vivre cela », nous disait une mère.

L'oppression ici, c'est de croire que l'on est tout seul et qu'on ne peut rien faire d'autre que d'espérer que ça aille mieux; c'est de croire que seul le pouvoir médical et institutionnel peut apporter soulagement à nos misères; $c^{\prime}$ est de se sentir petit, impuissant et surtout coupable de ce qui nous arrive. L'oppression, c'est aussi de se croire ignorant par rapport à la maladie que vit son enfant (même si ça fait de nombreuses années que l'on vit le problème) et de penser que le savoir ne peut nous être transmis que par " ceux qui savent ", en l'occurrence les spécialistes en la matière.

Pour les personnes atteintes de maladie mentale, être opprimé, c'est d'abord se sentir envahi, possédé par ses symptômes et ne plus penser pouvoir faire autre chose que de se replier sur soi-même. C'est, par exemple, sortir un soir et n'être pas capable d'engager une conver- 
sation ou même d'établir un contact avec une autre personne, c'est continuellement se retrouver seul. C'est, pour plusieurs, avoir envie d'affection, d'amour, vouloir vivre sa sexualité et être privé de tout cela. C'est devoir se plier aux soins médicaux et aux services sociaux pour pouvoir supporter le quotidien et, aussi, être supportable pour les autres.

Rarement, une personne atteinte de maladie mentale va accepter avec joie de se faire hospitaliser, de prendre une médication dont les effets secondaires à long et à court terme peuvent parfois être tout aussi dommageables que bienfaisants.

Être aux prises avec la maladie mentale, particulièrement dans notre région où parfois le taux de chômage dépasse les 20 pour 100 , c'est être confiné à vivre plus souvent qu'autrement de l'aide sociale. C'est porter, pour ceux qui s'y résignent, l'étiquette " d'inapte au travail " tout en nourrissant le rêve d'avoir un jour l'emploi qui leur permettra d'accéder à une vie "normale".

Souvent, la personne ne peut même pas gérer seule son chèque d'aide sociale. Si elle vit en famille d'accueil ou dans sa propre famille, elle finit par s'installer dans une dynamique d'assistance et parfois de surprotection où ce sont les autres qui pensent et agissent à sa place; où le quotidien très souvent se résume à dormir, à se bercer tout le jour en grillant cigarette sur cigarette et en dérangeant le moins possible.

Pour ceux qui vivent en appartement, il est parfois nécessaire d'avoir de l'aide pour gérer leur argent, car dès le premier jour du mois il pourrait ne plus rien leur rester pour payer la nourriture. Certains, en effet, par anxiété très fréquemment, vont être portés à dépenser de toutes sortes de façons l'argent qu'ils ont en main et cela, sans penser aux nécessités du lendemain; car demain fait souvent partie d'une réalité lointaine et confuse à leurs yeux.

L'oppression, c'est surtout de vivre des choix qui ne sont pas les siens. Ce peut être le travail dont on ne peut suivre le rythme et qui est source de grande anxiété, mais qu'on exécute " pour avoir un plus gros chèque " ou encore pour se plier au plan de l'institution soignante. Ce peut être de vivre en famille d'accueil, sans réel chez-soi et penser ne jamais pouvoir avoir sa propre vie avec son indépendance, son autonomie. C'est, bref, être conduit par les autres qui décident pour soi et ne plus se sentir qu'un objet de soins et de services.

Pour les parents comme pour les personnes atteintes de maladie mentale, la vie devient encapsulée à l'intérieur d'un espace où la dynamique créée par la maladie, ou qui a nourri cette maladie, prend toute la place et finit par ne plus laisser d'air respirable à personne.

C'est sur cette base, en s'appuyant sur cette réalité que les trois intervenants effectuant le suivi à l'externe des patients en psychiatrie ont convenu d'entreprendre une démarche qui aurait comme préoc- 
cupation première de laisser à ces personnes l'occasion de prendre leur place, de devenir des acteurs, des sujets agissant sur les éléments qui les oppriment et non plus les objets dociles des bons soins et services des pouvoirs institutionnels ou autres.

C'est le propre d'une démarche qui se veut, à notre avis, conscientisante.

\section{Une démarche conscientisante}

Une telle perspective exige que l'on fasse confiance aux forces qui résident dans chaque personne. Le propre d'une démarche conscientisante est dans les rapports horizontaux qui se tissent entre ceux et celles qui désirent cheminer ensemble.

Éducateurs et éduqués, orientés ensemble vers la réalité, se rencontrent dans une tâche dans laquelle les deux sont sujets agissant non seulement pour déchiffrer cette réalité et donc la connaître avec un esprit critique, mais aussi pour la recréer. (Freire, $1982: 49$ )

Paulo Freire fait référence, ici, à cet élément fondamental qui doit guider toute démarche de ce genre. Mais établir un rapport horizontal entre personnes, être véritablement ensemble, cela ne va pas de soi; chacun étant plus ou moins imbu de l'idée que le pouvoir, c'est l'autre qui en est le possesseur. On a intégré par sa culture que le savoir n'est pas à la portée de tout le monde et que ceux qui y ont accès sont justement ceux-là par qui les solutions doivent venir. En somme, chacun a un peu appris à acquérir les attitudes qui contribuent à le maintenir dans un état d'infériorité et objet de bienveillance. Ainsi, inconsciemment, chaque personne devient le principal agent de sa propre oppression.

Le moyen privilégié pour atteindre ce rapport horizontal entre les êtres, $c^{\prime}$ est le dialogue. Par le dialogue, chaque personne découvre la réalité de l'autre et prend peu à peu conscience qu'elle n'est plus seule à vivre telle ou telle situation. Ce partage qui donne la parole à qui veut la prendre et qui permet l'écoute fait également prendre conscience à chacun qu'il est possesseur d'un certain savoir en même temps que d'un certain pouvoir.

Le dialogue, c'est le ciment qui crée l'unité dans un groupe et qui finit par faire apparaître la solidarité. Ensemble, les personnes se rendent compte de leur force et apprennent peu à peu à l'utiliser afin d'agir elles-mêmes sur la réalité qui les opprime. D'objets de la bienveillance des autres, elles commencent à devenir des sujets agissants.

Une approche fondée sur le dialogue exige donc que chacun soit accepté avec sa réalité et avec le niveau de conscience qu'il en a. II faut partir de là où est la personne, et ce sera la somme de toutes ces réalités 
et de tous les niveaux de conscience qui créera l'ensemble. Ainsi, à partir des échanges entre les participants à un dialogue, les animateurs verront à dégager des thèmes qui seront générateurs d'autres thèmes et qui feront en sorte que la réalité prendra un autre visage. Elle pourra, de la sorte, devenir plus accessible à un changement.

Prenons comme exemple les parents dont nous avons parlé antérieurement et qui se sentaient complètement dépassés par la situation et totalement impuissants à y changer quoi que ce soit. En partageant leur expérience avec d'autres personnes vivant le même problème, ils pourront se rendre compte, peu à peu, d'abord qu'ils ne sont pas seuls dans leur cas; ainsi le poids du fardeau qu'ils portent leur sera déjà moins lourd. Ils prendront en plus conscience qu'à travers leur expérience, ils ont acquis des connaissances sur la maladie mentale, ils ont développé des stratégies pour agir dans les moments les plus difficiles. Ainsi ils se rendront compte de leur capacité à changer la réalité. Une capacité qui sera enrichie de l'expérience des autres.

En plus, à travers le dialogue, les personnes prendront conscience de leur force comme groupe. Les problèmes mis en évidence dans les réflexions deviendront des défis à relever, des " faire possible " et non plus des obstacles infranchissables.

Par la réflexion sur les problèmes vécus de chacun, la conscience qui percevait les choses de façon magique est appelée à devenir peu à peu critique; car c'est en établissant une distance entre soi et le problème que celui-ci commence à être perçu dans ses vraies dimensions. Ce qui en est la cause peut maintenant être vu avec plus de clarté et les actions à réaliser pour en modifier les effets sur sa vie deviendront plus perceptibles.

Avoir une conscience magique c'est, par exemple, penser que tout ce qui nous arrive est indépendant de nous, qu'il n'est que l'effet du hasard et les moyens pour changer cela sont ailleurs, chez ceux qui possèdent le savoir. C'est croire, comme cette personne qui s'exprimait au cours d'une rencontre de groupe, que la maladie mentale est une "épreuve du bon Dieu » et que seules les prières et les pilules pourront y changer quelque chose.

Le dialogue fait s'interroger ce type de conscience, le place devant la réalité et l'amène à grandir, à se développer. Mais la conduite d'une démarche dialogique doit tenir compte des différences culturelles, des positions de classe dans un groupe. II sera facile, pour un intellectuel habile à parler et à jouer avec des concepts, de prendre la tête d'un groupe où les membres viennent de toutes les couches de la société. Les personnes moins instruites, plus imbues de la vision magique des choses auront vite fait de se laisser impressionner par quelqu'un qui "parle bien » et qui a l'air de tout savoir. Ainsi, quelques-uns décideraient de la réalité à changer et mèneraient habilement le mou- 
vement en s'assurant que l'ensemble des membres suivent la marche. Cela est un peu le propre de nos approches traditionnelles en intervention communautaire aujourd'hui. II n'est sans doute pas facile d'aller au rythme de tout le monde. Le mouvement est beaucoup plus lent et moins spectaculaire, mais à mon avis, il est plus durable et plus vrai.

Cela demande aux animateurs d'être vigilants par rapport à de telles situations, de les prévenir en trouvant, avec tous les membres du groupe, les moyens qui incitent le plus au dialogue. Il s'agira de trouver les règles de fonctionnement qui feront consensus et l'animateur, quant à lui, aura comme rôle d'aider le groupe à les suivre. De la sorte, l'intervenant n'est pas le leader qui sait plus que les autres et guide la démarche. Il est celui qui accompagne et aide les personnes à faire de leurs réflexions des sources de lumière pour guider l'action.

\section{Application pratique}

\section{Formation d'un groupe de parents}

Depuis plus d'un an, nous étions trois intervenants à effectuer un suivi dans la communauté auprès de personnes souffrant de troubles mentaux sévères; cela se faisait à l'intérieur du programme de case management mis sur pied par le service de santé mentale du Centre hospitalier Baie-des-Chaleurs.

Nous avions alors eu à intervenir beaucoup auprès des parents : les soutenir, les aider à comprendre la maladie de leur enfant, faciliter les liens entre eux et le monde hospitalier; bref, nous étions en contact très étroit avec eux.

Nous pensions donc connaître leurs besoins par rapport à la maladie mentale; nous savions qu'ils désiraient avoir plus d'information, et surtout être écoutés, ne plus se sentir seuls. Déjà, disaient-ils, l'intervention que nous faisions les aidait beaucoup; nous avons cependant convenu d'aller plus loin.

Nous avons pensé leur proposer des rencontres où ils auraient l'occasion de se parler. Mais ces rencontres, nous avons pensé les faire en fonction de leur propre réalité. C'est ainsi que nous sommes d'abord allés dans les familles (nous en avons rencontré vingt-cinq) leur demander de nous raconter, à l'aide d'un questionnaire, leur vécu par rapport à la maladie de leur enfant. Les thèmes traités concernaient leur expérience antérieure et actuelle avec leur enfant, avec l'entourage, avec le milieu psychiatrique. Nous leur avons demandé d'exprimer leurs commentaires, leurs critiques quant à la façon dont ils avaient été traités par le milieu institutionnel, pour, enfin, leur demander de nous exprimer s'il y avait de leur part une motivation à changer quelque chose à leur vécu et, si oui, de suggérer les moyens. 
Cette collecte de données nous a fourni une somme considérable de thèmes qu'il nous a fallu mettre en ordre et avec lesquels nous avons constitué des tableaux codés. Ces tableaux reprenaient mot à mot les propos des parents de telle sorte qu'ils étaient la représentation exacte de ce qui avait été exprimé par eux. Nous avions divisé ces tableaux en deux grandes parties, c'est-à-dire ce qui avait été vécu dans le passé et ce qui se vivait actuellement.

Voici quelques réalités exprimées par les parents et transcrites sur ces tableaux : " misère à croire qu'il était malade ", "culpabilité ", " solitude ", " peur de le voir en appartement ", " il a fallu se battre pour se faire écouter ", etc.

Ces mots et ces expressions que nous avions recueillis, il fallait maintenant les redonner aux parents. Nous les avons alors invités à une rencontre (seize personnes sont venues) où ils ont pu prendre connaissance du résultat de notre démarche auprès d'eux. Ils ont pu constater qu'ils n'étaient pas seuls à vivre de telles situations et ont pu échanger leurs points de vue avec d'autres personnes dans le même cas.

Par des travaux en équipes puis en grand groupe où il fallait faire une synthèse des réalités exprimées sur les tableaux, où la démarche devait conduire à définir un problème commun et à déterminer une action commune (il a fallu huit rencontres de trois heures), les personnes sont passées peu à peu d'une perception individuelle de leur situation à une perception collective.

Sur les tableaux, chacun s'était reconnu dans ses propres paroles, mais aussi dans celles des autres. Par le partage de ces paroles, par le dialogue, prenait naissance, à travers le dévoilement de la réalité, une solidarité qui allait permettre au groupe de naître et devenir une force capable de transformer cette réalité.

Peu à peu, à travers ce travail de décodage, les personnes se sont dévoilées aux autres, elles ont exprimé leurs forces ainsi que leurs limites et, ensemble, elles se sont donné les moyens d'agir individuellement et collectivement pour changer des situations définies par elles comme étant opprimantes.

$C^{\prime}$ est ainsi qu'un parent qui se sentait incapable de prendre de la distance par rapport à son enfant, qui se laissait complètement envahir par la situation a pu se donner la force d'agir pour se procurer plus d'espace vital. Avec l'encouragement des autres personnes qui avaient vécu la même expérience et en faisant une analyse de la situation, il a pris conscience de ses propres forces.

Aussi, une mère a décidé de faire un travail dans sa propre famille, afin que son fils soit plus accepté par les oncles, tantes, cousins et cousines qui avaient l'habitude de venir à la maison. Cette mère a organisé ce qu'elle a appelé « des dîners-causeries » en compagnie de son enfant malade et des membres de la famille élargie. 
Il faut noter ici qu'une partie importante de chaque rencontre est consacrée au partage du vécu. Certains ont eu à vivre des moments encourageants durant le mois (nous avons une rencontre chaque mois); leur enfant a repris le travail ou encore, c'est un nouvel essai scolaire qui semble vouloir réussir. D'autres, par contre, ont dû traverser une crise très pénible où leur enfant a été hospitalisé et où les espoirs d'une " guérison " se sont presque anéantis.

$C^{\prime}$ est dans ces moments de partage que chaque personne va puiser un peu de force pour continuer à agir et où la solidarité prend naissance et se renforce.

Cette prise de conscience de ses propres forces a un effet également sur le groupe. C'est elle qui amène des personnes à se reconnaître une parenté et qui fait naître l'unité. De la sorte, les participants à cette démarche ont réalisé qu'ils pouvaient changer des choses ensemble par rapport à certains problèmes relevés. Ils ont décidé d'abord de faire un travail de sensibilisation auprès de la population, afin de faire mieux connaître le vécu des parents aux prises avec la maladie mentale. Ils ont participé à une chronique dans les journaux locaux, à la radio, ainsi qu'à des activités de formation populaire. Afin de transformer les rapports entre le milieu institutionnel et les familles, ils ont organisé une rencontre-dialogue avec les intervenants en santé mentale du Centre hospitalier Baie-des-Chaleurs où il a été possible de jeter les bases d'un partenariat entre les parents et les intervenants.

En se transformant peu à peu en acteurs à travers un long processus de prise en charge de leur propre réalité, ces personnes sont en train de se sortir de cet état de dépendance et d'impuissance par rapport à ce qui les opprimait. Elles se sont mises en marche. L'important, ici, n'est pas d'atteindre le but ultime, mais d'aller dans sa direction.

\section{Formation d'un groupe de personnes souffrant de maladie mentale}

Un an après la mise en place du programme de case management, nous pensions qu'une intervention de groupe avec les personnes dont nous faisions le suivi serait un heureux complément à l'intervention individuelle.

Nous avions pour but, en les regroupant, de leur permettre de se rencontrer et, ainsi, de couper leur isolement. Mais contrairement aux parents avec qui nous avions utilisé le dialogue en groupe pour faire naître l'unité, avec eux nous avions plutôt décidé de passer par des expériences de loisir où peu à peu, de façon informelle, chacun serait amené à partager son vécu avec quelqu'un d'autre. 
Notons ici que les personnes atteintes de schizophrénie ou qui sont aux prises avec des problèmes psychotiques importants n'ont pas cette capacité $d^{\prime}$ introspection qui leur permet de prendre contact avec leurs émotions et d'en parler ouvertement.

C'est justement le propre du psychotique de dévier dans un monde irréel plutôt que d'avoir à sentir ses émotions. Elles sont pour lui source de souffrances intenables et c'est pourquoi il ne s'y donne pas accès.

Dans ces conditions, il était inutile pour nous de penser à amener ces personnes à réfléchir ensemble sur leur vécu; nous avons plutôt pensé qu'à travers des projets préparés et réalisés ensemble, des liens allaient se tisser entre elles et, ainsi, donner naissance à une certaine forme d'unité.

$C^{\prime}$ est ainsi que la première année nous avons vécu diverses expériences, telles qu'une excursion près d'une rivière pour observer les saumons, un voyage à Percé, nous sommes allés faire de la raquette, jouer aux quilles, manger au restaurant, etc. Chacun de ces projets était précédé d'une rencontre de préparation et suivi d'une évaluation par le groupe.

Ainsi, à l'occasion des activités, plusieurs personnes avaient créé des liens significatifs, s'étaient parlé de leurs séjours en milieux hospitaliers, de leur médication, avaient en somme commencé à se révéler à I'autre.

Au cours des rencontres d'évaluation, la possibilité leur était donnée de revenir sur ce qui s'était vécu en groupe, d'en parler ouvertement. Ces rencontres d'évaluation nous permettaient à nous, les animateurs, de vérifier les alliances que nous avions pu observer, ainsi que d'autres constatations faites au moment des activités.

De cette façon, chacun avait le loisir de s'exprimer à sa guise et de prendre la place qui lui convenait dans le groupe.

Nous nous trouvions cependant toujours placés devant la réalité de ces personnes qui ont de grandes difficultés (pour la plupart cela paraît impossible) à livrer leur vécu antérieur, à partager, en somme, ce qui est souffrance en elles. L'angoisse, l'anxiété, leur pensée souvent éclatée créent parfois un blocage à la communication qui est dialogue et qui fait naître la solidarité.

Nous avons donc pensé que par des moyens créateurs et libérateurs de l'esprit, il aurait été possible de se révéler aux autres sans se sentir menacé.

Nous pensions que l'expression artistique par le dessin ou la peinture, par exemple, allait leur permettre de réaliser cela. L'idée était de les amener à s'exprimer, à parler d'eux par ces moyens, mais sans avoir à expliquer l'objet de leur expression et sans qu'ils aient à craindre de subir quelque interprétation ou jugement de la part des autres. Nous avions foi en la capacité libératrice de l'expression artistique. 
C'est ainsi que depuis deux ans maintenant, nous nous sommes associés à une artiste-peintre qui a eu le rôle d'animer des ateliers de créativité.

Nous, les trois "case managers", sommes devenus des participants au même titre que les autres en ce qui a trait au travail en atelier et c'est l'animatrice qui a eu la responsabilité de guider le groupe dans sa démarche artistique.

Nous avions prévu une rencontre de trois heures par semaine avec tout le groupe et une fois par mois une réunion d'évaluation et d'orientation entre les trois intervenants et l'animatrice.

L'objectif général que nous avions alors énoncé était le suivant : Susciter chez les personnes un sentiment $d^{\prime}$ appartenance à un collectif et créer un élan de solidarité capable de les amener à prendre une part plus grande, individuellement ou collectivement, aux réalités qui les concernent.

Les objectifs particuliers se lisaient comme suit :

- Permettre aux personnes de créer des liens et de quitter leur isolement.

- Voir à créer une force de groupe qui stimule pour le reste de la semaine.

- Augmenter l'estime de soi.

- Se faire reconnaître socialement par le groupe.

- Venir chercher des habiletés sociales en vue d'une réinsertion sociale.

- Apprendre à se faire des amis et à les garder.

- Apprendre à être constant dans sa participation.

- Apprendre à être heureux dans un travail, à ne pas le voir comme une corvée.

- Connaître la sensation de stabilité émotive à travers un groupe.

Nous en sommes maintenant à notre troisième année d'expérience en atelier. Après la première année, nous constations que la participation avait été excellente (une quinzaine de personnes venaient régulièrement), que contrairement à ce que l'on aurait pu croire les personnes avaient réussi à travailler avec une grande concentration et nous avions noté qu'à mesure que la démarche progressait, s'installait un esprit de corps, d'unité dans le groupe où chacun se sentait de plus en plus solidaire de l'autre.

Les commentaires des participants au moment d'une rencontre d'évaluation étaient très éloquents à cet effet : « le groupe était chaleureux ", " on a pu se faire des amis ", " on ne s'est pas senti jugé, il n'y avait pas de préjugés ", etc.

C'est ainsi qu'après nous être rendu compte que le groupe était de mieux en mieux structuré, cimenté, nous avons convenu qu'il était 
nécessaire de donner un caractère permanent à cette expérience. II ne fallait surtout pas interrompre un processus dont la réussite commençait à se vérifier à travers le vécu quotidien, que ce soit sur le plan individuel ou collectif.

Certains se disaient plus confiants par rapport à leur entourage. Le fait de sortir de chez eux les faisait en quelque sorte sortir de leur carapace pour communiquer avec les autres.

Au moins cinq membres du groupe, à un moment donné, étaient en processus de travail ou de retour aux études. Nous pensions que I'intervention de groupe avait pu être un élément dynamisant pour ces personnes.

La deuxième session, qui s'est déroulée de novembre 1989 à juin 1990, nous a amenés sensiblement aux mêmes conclusions et a confirmé chez nous l'idée que la force née d'un collectif, par la solidarité qui s'y crée à travers les échanges et les expériences qui s'y vivent, est l'élément moteur de tout changement susceptible de s'opérer chez une personne. Parce qu'il implique la personne avec les autres et que ce sont elles, ensemble, qui sont les acteurs premiers de ces changements. Le groupe devient alors source d'énergie.

Au cours de cette session, nous avons pu voir le groupe se consolider par des expériences en atelier et des projets tels une journée passée ensemble dans la maison d'un intervenant, couronnée par un souper où chacun a dû amener sa participation, un voyage planifié et vécu en groupe, une journée à la cabane à sucre, des moments de discussion, de partage et de création (composition d'une chanson sur le thème : "Vivre sa différence ») où chacun a pu prendre la place et la part qui lui convenaient.

Bref, cette deuxième partie de l'expérience de créativité nous a amenés à la conviction de plus en plus forte que, pour que reste ferme ce sentiment d'appartenance au groupe et que ce dernier demeure source de solidarité transformatrice, il est nécessaire d'ancrer la démarche dans un lieu précis appartenant à ce groupe. C'est ainsi que l'idée de se trouver un local (maison ou appartement) où chacun se sentirait chez lui a pris naissance et s'est propagée dans le groupe.

Depuis maintenant six mois, nous avons notre propre local dans la communauté (auparavant nous allions au local de l'animatrice) et c'est là que nous tenons nos ateliers. Le groupe, quant à lui, prend de plus en plus corps. Plusieurs personnes, en dehors des activités planifiées, maintiennent des liens d'amitié (certaines liaisons amoureuses ont même vu le jour). En somme, nous pouvons dire que notre démarche avec ce groupe pour lequel nous avons choisi d'expérimenter la création artistique jumelée à des activités de loisir nous aura permis d'amener les personnes qui en font partie sur la voie de l'unité et de l'agir. Agir sur sa personne et agir aussi ensemble sur ce qui nous concerne 
tous. Dans la programmation pour l'année 1990-1991, les personnes ont décidé d'inclure une discussion avec le psychiatre de l'hôpital au sujet de la médication. Elles ont le goût d'échanger avec lui à propos de leur vécu par rapport à cette réalité et désirent être mieux renseignées.

Ainsi, dans cette programmation, les ateliers d'art sont jumelés à des activités de loisir et d'information et à des discussions sur des sujets qui concernent chaque participant (alimentation, sexualité, médication), de telle sorte que peu à peu leur horizon s'ouvre et laisse paraître une réalité un peu moins menaçante.

De personnes captives de leurs angoisses et de leurs délires, elles deviennent, à très petits pas, ouvertes à une nouvelle façon de voir et de faire le monde.

\section{Conclusion}

Une démarche qui se veut conscientisante exige au préalable une remise en question de l'approche institutionnelle. Une approche où la plupart du temps, on ne fait qu'appliquer des politiques gouvernementales dans le simple souci très professionnel de donner les meilleurs soins et services auxquels la personne a droit. Une approche basée sur notre modèle politique où quelques-uns décident et $d^{\prime}$ autres exécutent; où certains donnent et d'autres reçoivent.

Ainsi, nous devons remettre en question notre modèle professionnel, corporatiste, à travers lequel l'intervenant est appelé à développer une attitude qui l'amène à être distant des problèmes auxquels il fait face. Il est le savoir, il est le pouvoir, il est ce sauveur vers qui l'on accourt lorsqu'on est mal pris.

Adhérer à une perspective conscientisante en tant qu'intervenant professionnel, $c^{\prime}$ est accepter de descendre de ce piédestal où l'on est monté plus ou moins consciemment à travers la formation acquise et les rôles que l'on a accepté de jouer. C'est renoncer au cadre hiérarchisé imposé par notre modèle politique et accepter de marcher côte à côte avec les gens dont les problèmes nous préoccupent. C'est cesser d'être pour, pour peu à peu, au fil de l'expérience, être avec.

Cette attitude qui place l'intervenant avec les personnes le situe dans une position où, avec elles, il ira dans son action, bien au-delà de l'attribution de soins et de services. II sera cet animateur-participant, engagé avec les gens dans la transformation du monde.

Les chefs de file de cette vision des choses au Québec, actuellement, sont les groupes communautaires dits alternatifs. Alternatifs, justement parce qu'ils apportent une autre vision de l'intervention avec les personnes souffrantes. 
Avec le peu de moyens dont ils disposent et souvent boudés par les milieux institutionnels, ils ont su mettre en question nos visions professionnelles et se présenter sur la scène de l'intervention psychosociale en acteurs essentiels à l'amorce d'un changement dans notre vision collective de voir les choses. La politique de la santé mentale est, à sa mesure, un exemple du changement de mentalité qui s'opère, par la reconnaissance de ces groupes comme partenaires " invités à participer étroitement à l'organisation régionale de nos services " (Ministère de la Santé et des Services sociaux, gouvernement du Québec, Politique de la santé mentale, 1989, p. 50), reconnaissance acquise à force de patience et d'acharnement de leur part.

II est facile, dans une intervention de groupe, de reproduire le modèle institutionnel hiérarchisant où quelques-uns, plus sensibilisés à leurs droits et à ceux des autres, vont devenir les leaders très actifs d'un ensemble de personnes qui leur laisseront à eux le rôle de répondre en leur nom. Ainsi, un groupe peut paraître très actif et dynamique, mais l'action n'est souvent l'œuvre que de quelques personnes.

Une action issue d'un modèle vertical, hiérarchique peut, bien sûr, donner des résultats. Elle peut même en arriver à faire changer des politiques, mais à long terme, elle ne s'interrogera pas en profondeur sur les rapports de pouvoir dans la société, puisque son cadre d'intervention est le même que celui qui a créé les situations d'oppression. Une approche conscientisante se veut horizontale, chacun, peu à peu, à force de parole et d'écoute, se joignant à l'autre dans un lent mouvement de solidarité. Ainsi, le leadership naîtra du groupe lui-même et non pas de quelques personnes plus habiles que les autres.

Ici, ce ne sont pas les discours ni les actions d'éclat qui comptent, mais bien les petits gestes accomplis par l'ensemble des personnes concernées. Ces petits gestes, lorsqu'ils sont le fruit d'une plus grande conscience de la réalité dans ses multiples dimensions, sont alors porteurs d'un germe de transformation, de recréation du monde.

\section{Notes}

1 Un ouvrage portant sur le sujet a été écrit par l'auteur de cet article : Conscientisation et Santé mentale, Centre hospitalier Baie-des-Chaleurs, 1990, 153 p.

2 Paulo Freire est un éducateur brésilien qui, au début des années 60 , a élaboré et mis en pratique une méthode $d^{\prime}$ alphabétisation conscientisante pour les masses populaires du Brésil.

${ }^{3}$ Depuis six ans, existe au Centre hospitalier Baie-des-Chaleurs un programme de case management où trois intervenants effectuent un suivi des personnes atteintes de troubles mentaux sévères (psychose, schizophrénie) et vivant dans la communauté. 


\section{Références bibliographiques}

Freire, Paulo (1982). Pédagogie des opprimés, suivi de : Conscientisation et Révolution. Paris : Maspero, p. 49 (Petite Collection Maspero).

Ministère de la Santé et des Services sociaux (1989). Politique de la santé mentale. Gouvernement du Québec. 